$5-1996$

\title{
Behavior and Performance of Adsorptive Natural Gas Storage Cylinders During Discharge
}

\author{
K. J. Chang \\ Cleveland State University \\ Orhan Talu \\ Cleveland State University \\ Follow this and additional works at: https://engagedscholarship.csuohio.edu/encbe_facpub \\ Part of the Thermodynamics Commons
}

How does access to this work benefit you? Let us know!

\section{Publisher's Statement}

NOTICE: this is the author's version of a work that was accepted for publication in Applied Thermal Engineering. Changes resulting from the publishing process, such as peer review, editing, corrections, structural formatting, and other quality control mechanisms may not be reflected in this document. Changes may have been made to this work since it was submitted for publication. A definitive version was subsequently published in Applied Thermal Engineering, [16, 5, (May 1996)] DOI 10.1016/1359-4311(95)00017-8

\section{Original Citation}

Chang, K. J., , \& Talu, O. (1996). Behavior and performance of adsorptive natural gas storage cylinders during discharge. Applied Thermal Engineering, 16(5), 359-374.

\section{Repository Citation}

Chang, K. J. and Talu, Orhan, "Behavior and Performance of Adsorptive Natural Gas Storage Cylinders During Discharge" (1996). Chemical \& Biomedical Engineering Faculty Publications. 64.

https://engagedscholarship.csuohio.edu/encbe_facpub/64

This Article is brought to you for free and open access by the Chemical \& Biomedical Engineering Department at EngagedScholarship@CSU. It has been accepted for inclusion in Chemical \& Biomedical Engineering Faculty Publications by an authorized administrator of EngagedScholarship@CSU. For more information, please contact library.es@csuohio.edu. 


\title{
BEHAVIOR AND PERFORMANCE OF ADSORPTIVE NATURAL GAS STORAGE CYLINDERS DURING DISCHARGE
}

\author{
K. J. Chang and Orhan Talu \\ Department of Chemical Engineering, Cleveland State University, Cleveland, Ohio 44115, USA
}

\begin{abstract}
Adsorbed natural gas (ANG) has the potential to replace compressed natural gas in mobile storage applications, such as in vehicles. Although a substantial research effort has been devoted to ANG, very few studies evaluate the impact of heat of adsorption on system performance. This paper concentrates on the impact of heat of adsorption on ANG performance during discharge, while the gas outflow rate is dictated by the energy demand of the application. The temperature drop and performance loss was measured with commercially available ANG cylinders under realistic conditions. Data show as high as a $37^{\circ} \mathrm{C}$ temperature drop at high discharge rate, with a performance loss approaching $25 \%$ of isothermal capacity. The performance loss is expected to be $15-20 \%$ at moderate discharge rates. Analysis of data and predictions of a simple model indicate that the ANG system is neither adiabatic nor isothermal during discharge; the thermal capacity of the vessel wall and external heat transfer conditions have a significant effect on system behavior. The poor thermal conductivity of packed adsorbent is a major obstacle for the utilization of these energy sources. Changing the flow direction during discharge from axial to radial by a perforated tube inserted at the center of the cylinder significantly reduces the performance loss by increasing the heat transfer from the wall to the central region. At intermediate discharge rates, where the inserted tube has the largest impact, the performance loss is reduced to $12 \%$ from $22 \%$ without the tube under identical conditions.
\end{abstract}

Key words-Adsorption, natural gas, storage, methane, adsorption heat.

\section{NOMENCLATURE}

$\begin{array}{ll}b_{0}, b_{1} & \text { virial isotherm constants } \\ C_{\mathrm{c}} & \text { carbon heat capacity } \\ C_{\mathrm{p}} & \text { methane heat capacity } \\ C_{\mathrm{s}} & \text { steel heat capacity } \\ D & \text { cylinder diameter } \\ f & \text { molar flux at the top of the packing } \\ F & \text { total volumetric flow out of the cylinder } \\ h & \text { natural convection heat transfer coefficient } \\ k_{0}, k_{1} & \text { Henry's law constants } \\ L & \text { length/height of the cylinder } \\ N & \text { amount adsorbed } \\ P & \text { pressure } \\ Q & \text { total molar flow rate out of the cylinder } \\ r & \text { radial position } \\ R & \text { cylinder radius } \\ R_{\mathrm{B}} & \text { universal gas constant } \\ t & \text { time } \\ T & \text { temperature } \\ T_{\text {amb }} & \text { ambient/charge temperature } \\ <T> & \text { volume average temperature } \\ \Delta T & \text { temperature difference } \\ <\Delta T> & \text { volume average temperature drop } \\ v & \text { specific volume of gas } \\ V & \text { total cylinder volume } \\ V \text { per } V & \text { performance parameter, see equation }(10) \\ W & \text { steel wall thickness } \\ z & \text { axial position } \\ G r e e k \text { letters } & \\ \epsilon & \\ \rho_{\mathrm{c}} & \text { total packing porosity } \\ \rho_{\mathrm{s}} & \text { carbon density } \\ \kappa & \text { steel density } \\ \tau & \text { effective packing conductivity } \\ \text { dimensionless time }\end{array}$




\section{INTRODUCTION}

Natural gas (NG) is an abundant, clean-burning fuel. Its major use at present is for combustion in stationary applications, such as space heating. Mobile applications are limited, since the energy density of stored natural gas is low due to its gaseous nature. Natural gas is a mixture comprised mainly of methane, some low hydrocarbons and some inert gases. It cannot be liquified at ambient temperature. Nevertheless, it has a perceived potential to replace liquid fuels in the future, due to geopolitic and environmental reasons. Vehicles operating with compressed natural gas (CNG) already number about $1,000,000$ worldwide [1].

Compressed natural gas systems operate at high pressure to enhance the storage density, which increases the cost and represents a safety concern [2]. An alternative to CNG is adsorbed natural gas (ANG) storage, where a microporous solid (i.e. activated carbon) is packed in the vessel to increase the storage density, enabling lower-pressure operation with the same capacity. Considerable research effort has been devoted to ANG storage; fundamental molecular simulations [3-6], materials development and characterization [7-12], simulation of dynamic behavior [13-15], experimental measurements of performance under dynamic conditions [16-18], feasibility studies [19] and concept demonstrations [20]. A fairly comprehensive review of the technology and its current state is provided by Talu [2].

Adsorption is an exothermic process. Any finite rate of adsorption (or desorption) is accompanied by temperature changes in an ANG storage system. The heat of adsorption has a detrimental effect on performance during both charge and discharge cycles. A temperature increase as high as $80^{\circ} \mathrm{C}$ has been measured [18] and modeled [14] during the charge cycle. Although the temperature increase during the charge cycle is important, we do not believe it is critical for mobile ANG applications due to two reasons: (1) the charge cycle will be normally performed in a fuel-station where the necessary hardware can be built to remove the released heat of adsorption if a 'fast' charge is necessary; and (2) the highest perceived potential for mobile ANG is for fleet vehicles to be charged at a central site over a long period (i.e. overnight) which provides enough time for 'slow' charge to dissipate the heat of adsorption to the surroundings.

Contrary to the charge cycle, the rate of discharge is dictated by the energy demand of the application. Discharge time cannot be widely varied to moderate the impact of cooling during the use of ANG cylinders. It is also not feasible to include excessive hardware to moderate the temperature drop in a mobile application. This study concentrates on the impact of heat of adsorption during ANG discharge, which may play a crucial role in determining the feasibility of mobile applications.

As natural gas is discharged from an ANG system, the vessel cools down due to the heat of desorption. As a result, a larger amount of gas is retained in the system at the depletion pressure compared to isothermal operation, see Fig. 1. At any finite discharge rate, the amount of gas delivered under dynamic conditions is always lower than isothermal operation.

In this work, the behavior of ANG systems during discharge is thoroughly investigated with extensive experimental data collected under realistic conditions, as described in the following section. A simple, yet effective, model is developed to predict the behavior and to quantify properties which are not readily measurable. The time transients of important properties during the discharge of ANG systems are demonstrated with data and model predictions. The analysis of results leads to a simple remedy to moderate the penalty paid to the heat of adsorption. The benefits of this remedy are shown with experimental measurements in this paper, modeling and optimization of the idea are currently being performed.

\section{EXPERIMENTAL}

Two experimental systems were used: (1) high-pressure Cahn balance to measure the adsorption isotherms by standard gravimetric technique; and (2) a custom-built ANG test system. In addition, 


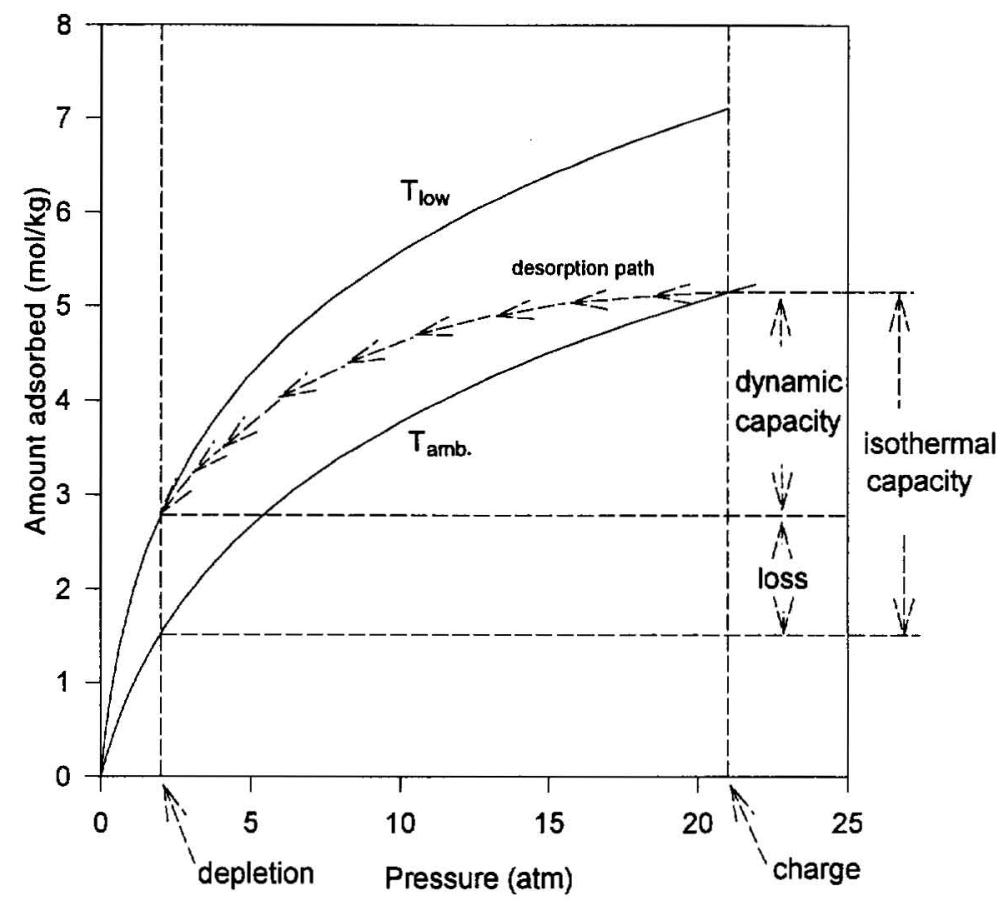

Fig. 1. Illustration of the impact of heat of adsorption on capacity during discharge of ANG systems.

some bench-top experiments were performed to determine packing densities and particle size of the carbon. Technical grade methane $(99 \%)$ was used in the experiments instead of natural gas.

The majority of the experiments were performed with the ANG test system shown in Fig. 2. The apparatus consisted of two main parts: (1) the control unit; and (2) the test cylinders. Charge/discharge rates and pressures were varied in the control unit, which also included a gas meter and a recorder/display for the thermocouples. The test cylinders were equipped with 12 thermocouples distributed throughout the volume and 2 thermocouple pads on the outside surface.

It was decided to use 'real' cylinders in this work to prevent any bias in the dynamic response of the system. Small, lab-scale test cylinders usually have an unrealistically high tare weight to carbon weight ratio, which biases the measured trends. Commercial-size ANG cylinders were obtained from G-Tec Co., which markets them for welding applications. These were regular carbon-steel propane cylinders filled with an activated-carbon adsorbent. As such, the cylinder walls were not suitable for drilling holes for thermocouple access, which was unavoidable for our purposes. The safety concern raised by the weakened mechanical strength of the cylinders was circumvented by placing them in a certified 'explosion room.' The control unit was outside the explosion room and safely accessible by the operator.

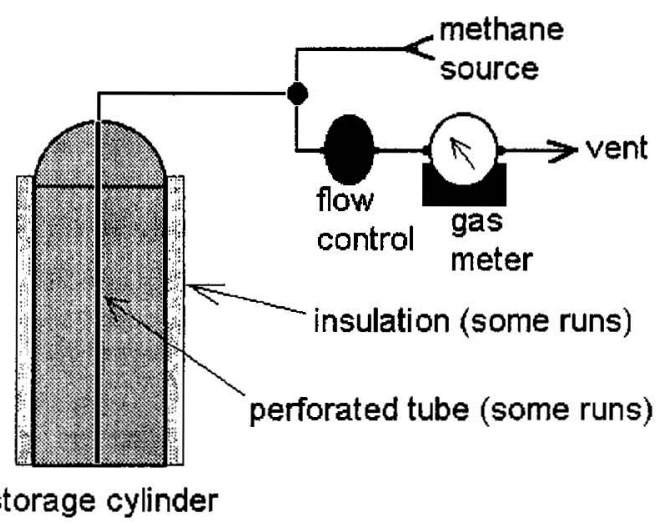

Fig. 2. Schematic of dynamic ANG test system. 
The main experimental variation was the discharge flow rate. It was varied between 1 and 15 $1 / \mathrm{min}$ at ambient conditions, 6-7 $1 / \mathrm{min}$ corresponds to the demand rate per cylinder on a subcompact car with 4 cylinders traveling at cruising speed. Two cylinders with different geometries were used in the experiments. Some experiments were performed with $10 \mathrm{~cm}$ fiberglass insulation wrapped around the cylinders to assess the importance of external heat transfer. As a final experimental variation, one of the cylinders was fitted with a perforated tube insert, as explained in detail later.

Experimental procedure involved overnight charge, where the cylinder was brought to 21 bar methane pressure and uniform temperature (ambient). A fixed, controlled rate discharge followed. The pressure, the amount of gas output and all thermocouple readings were recorded as a function of time during discharge. Experiments lasted from 60 to $1200 \mathrm{~min}$.

After the discharge cycle, the cylinder was closed and left to warm-up to ambient temperature. A final pressure was recorded when the temperature was uniform, which provided a check of experimental accuracy by the overall material balance. The difference between the amount of methane in the cylinder after warm-up and the initial amount at charge conditions should equal the total gas output measured during the discharge cycle. The amounts were calculated by the isotherm relation and the known packing density. The material balances were closed within $5 \%$ for all experiments.

\section{MODEL}

A comprehensive model for the dynamic response of ANG cylinders during discharge was solved by Sangani [13]. His results indicated that several phenomena had only incidental effects, which may be neglected under typical discharge conditions of ANG systems. In this work, we aimed at the simplest model which included all the essentials of the system. The dynamic model has two components: (1) the Equation of Continuity; and (2) the Equation of Energy. These two partial-differential equations are solved with the phase equilibrium relations, which include the effect of temperature on the isotherms and the variation of heat of adsorption with coverage.

\section{Equation of continuity}

Uniform pressure is assumed within the storage cylinder. Experimentally determined pressure gradients were usually less than 0.07 bar ( 1 psi, which was the accuracy of the pressure gage) and never exceeded 0.2 bar. The momentum equation becomes redundant once uniform pressure is assumed.

Instantaneous phase equilibrium is assumed between adsorbed and gaseous methane. The discharge process is slow enough to justify the neglection of any mass transfer resistance.

Based on the results of the prior rigorous modeling work, the bulk flow is assumed to be only in the axial direction, there is no radial flow in the model. The very small flow generated by natural convection within the cylinder does not have any significant impact on the overall results. A similar conclusion was drawn by Mota [14] in a theoretical modeling study of ANG systems.

Finally, gas-phase accumulation is neglected during the solution of the model, but it is included in the results. Rather than including the gas-phase accumulation in the simulations, it is mathematically easier to solve the model at a demand rate which is reduced by the depletion in the gas phase. The demand rate was reduced by assuming a constant drop in pressure with time. Although the pressure drop is not linear in reality, the variation in $\mathrm{d} P / \mathrm{d} t$ is small. Furthermore, the gas-phase contribution never exceeds $15 \%$ of the total contents of the cylinder, which makes the overall impact of this assumption even smaller. Some of the simulation results with the reduced demand rate are directly comparable to data, i.e. temperature profiles. Other properties, such as dynamic efficiency are adjusted for gas contribution at the end of the simulation.

With the four assumptions: (1) uniform pressure; (2) instantaneous phase equilibrium; (3) only axial flow; and (4) no gas-phase accumulation, the equation of continuity is written for a cylindrical shell of infinitesimal radial thickness. All properties are uniform in the axial direction, variations exist in the radial direction because of the temperature gradient:

$$
-\epsilon f / L=(1-\epsilon) \rho_{\mathrm{c}} \mathrm{d} N / \mathrm{d} t,
$$


where $f$ is the molar gas-phase flux at the top of the packing, $L$ is the height of the differential element which equals cylinder height, $N$ is the amount adsorbed, $t$ is time, $\epsilon$ is the void fraction of the packing, and $\rho_{\mathrm{c}}$ is the carbon density.

Initially the bed is at charge conditions: room temperature $\left(18-21^{\circ} \mathrm{C}\right)$ and 21 bar. Exact experimental values were used in the simulations:

$$
t=0 ; N=f(T, P) \text { at charge. }
$$

The molar flux $(f)$ is determined by the imposed molar demand rate on the system $(Q)$. An integral boundary condition at the top of the cylinder relates the two quantities:

$$
-\epsilon \int_{0}^{R} f .2 \pi r \mathrm{~d} r=Q
$$

where $R$ is the cylinder radius.

\section{Equation of energy}

Two simplifications were adopted for the equation of energy. Uniform temperature was assumed in the axial direction, since the bulk flow is in the axial direction. This assumption is later compared and justified with data. Finally, the gas and solid temperatures were assumed to be equal at all locations in the packing, neglecting any heat transfer resistance between the gas and carbon particles. The equation of energy becomes

$$
-\frac{f}{L} C_{\mathrm{p}} \Delta T+\frac{\kappa \delta}{r \delta r}\left(r \frac{\delta \Delta T}{\delta r}\right)=\frac{(1-\epsilon)}{\epsilon} \rho_{\mathrm{c}}\left(C_{\mathrm{c}} \frac{\delta \Delta T}{\delta t}-\lambda \frac{\delta N}{\delta t}\right)
$$

where $C_{\mathrm{p}}$ is the heat capacity of methane, $\Delta T$ is temperature difference from ambient temperature, $\kappa$ is effective conductivity of the bed, $r$ is the radial position, $C_{\mathrm{c}}$ is the carbon heat capacity and $\lambda$ is the heat of adsorption.

The first term on the left-hand-side of equation (4) represents the sensible heat removed from the cylinder by the exiting methane flow. The second term is the conduction in the radial direction. The first term in parenthesis on the right-hand-side is the energy accumulation of the adsorbent and the second term is the energy necessary for desorption. It should be noted that equation (4) neglects the sensible heat of the gas phase in accordance with negligible accumulation assumption used with the equation of continuity. Simultaneously, the decompression work done by the gas is also neglected.

Initially, the cylinders were equilibriated with ambient temperature at charge pressure, the initial condition is

$$
t=0 ; \Delta T=0 .
$$

Centerline symmetry provides one boundary condition in the radial direction:

$$
r=0 ; \delta \Delta T / \delta r=0 \text {. }
$$

The second boundary condition in the radial direction is determined by the energy balance on the cylinder wall. The carbon-steel cylinder wall has a large thermal capacity, which cannot be overlooked in these systems. The cylinder wall is assumed to be at uniform temperature. Natural convection takes place outside the cylinder wall with the ambient air, unless the cylinder is insulated. Energy is transferred into the carbon packing by conduction at the inner wall. The wall boundary condition is

$$
r=R ;-\kappa \delta \Delta T / \delta r=W C_{\mathrm{s}} \rho_{\mathrm{s}} \delta \Delta \Theta / \delta t+h \Delta \Theta,
$$

where $W$ is the wall thickness, $C_{\mathrm{s}}$ is the steel heat capacity, $\rho_{\mathrm{s}}$ is the density of steel, $\Delta \Theta$ is the difference between the wall temperature and the ambient temperature and $h$ is the natural convection heat transfer coefficient expressed as a function of Grashoff and Prandtl numbers by a well-known correlation [21]. Note that energy transfer between the wall and the interior is by 
conduction only. Convective heat transfer may play a role at higher flow rates than those used in this study.

\section{Phase equilibrium relation}

The phase equilibrium relation is necessary to calculate the amount adsorbed and the heat of adsorption at any given temperature and pressure. The discharge from ANG cylinders under realistic conditions is mainly an 'equilibrium'-driven process. Therefore, we aimed at representing the phase equilibria as accurately as possible.

Isotherm data collected with the high-pressure Cahn balance were successfully correlated with the Virial isotherm equation (22) with only four temperature-independent constants. The isotherm equation is

$$
P=N \exp \left[\left(k_{0}+k_{1} / T\right)+\left(b_{0}+b_{1} / T\right) \cdot N\right],
$$

where $P$ is the pressure and $k_{0}, k_{1}, b_{0}$ and $b_{1}$ are temperature-independent parameters. All isotherm data at several temperatures were curve-fitted to equation (8) in a single regression step, as suggested by Talu et al. [22]. The parameter values are listed in Table 1. The data is very well represented by the model, as can be seen in Fig. 3.

The heat of adsorption is determined from equation (8) as a function of the amount adsorbed with the appropriate thermodynamic formulations as

$$
\lambda=-R_{\mathrm{g}}\left(k_{1}+b_{1} \cdot N\right),
$$

where $R_{\mathrm{g}}$ is the universal gas constant.

\section{Parameter values and the solution method}

The values of the parameters in equations (1)-(9) are listed in Table 1. Two cylinder geometries were considered. The demand rate was varied from 1 to $15 \mathrm{l} / \mathrm{min}$ methane discharge flow at ambient conditions. The packing density was determined experimentally. The isotherm data was curve-fitted as described. The rest of the parameters were estimated considering the nature of the carbon adsorbent and methane.

The model was first nondimensionalized. The Crank-Nicholson method was used to expand the partial derivatives in finite difference form [24]. The resulting equations are nonlinear and coupled. The solution at each time step was determined with the Newton-Raphson root-finding technique with analytical derivatives. The simulations take about 2 min on a $486 / 33$ machine compared to the full model, which took about $45 \mathrm{~min}$ on a Vax 8650 [13].

Simulations were started with the cylinder at the measured charge conditions and stopped when the pressure dropped below 1.66 bar when the cylinder was 'depleted'. The experiments were stopped at this pressure since it was not possible to control the discharge rate at lower pressures. A pressure differential of 0.66 bar above atmospheric pressure also seems to be reasonable during the operation of a vehicle to force the flow of NG from the storage cylinder to the engine. Note that a susbtantial amount of residual methane remains in the cylinder at depletion.

\section{PERFORMANCE MEASURES}

A large amount of data is generated in the experiments and by the simulations. It is necessary to develop some simple indicators of ANG performance. The most commonly used indicator of overall performance is a volume ratio defined as [2]:

$$
V \text { per } V=\text { discharge gas volume at ambient conditions } / V \text {, }
$$

where $V$ is the cylinder volume; $V$ per $V$ is also equal to the dimensionless time in the simulations defined as

$$
\tau=Q \cdot v \cdot t / V,
$$

where $Q$ is molar discharge rate, $t$ is time and $v$ is molar volume at ambient conditions.

The impact of heat of adsorption on overall performance is measured by dynamic efficiency, 


\begin{tabular}{l}
\hline Operational parameters \\
\hline$T_{\text {amt }}=18$ to $22 \mathrm{C}$ \\
$Q=1-151 /$ min \\
Cylinder geometries \\
$L=66$ and $74 \mathrm{~cm}$ \\
$R=13$ and $10 \mathrm{~cm}$ \\
Packing/carbon properties \\
$\epsilon=0.5$ \\
$\rho_{\mathrm{c}}=0.975 \mathrm{gm} / \mathrm{cm}^{3}$ \\
$\kappa=2.12 \mathrm{E}-3 \mathrm{~J} / \mathrm{cm}^{3} / \mathrm{s} / \mathrm{K}$ \\
$C_{\varepsilon}=1052 \mathrm{~J} / \mathrm{kg} / \mathrm{K}$ \\
Steel wall properties \\
$W=0.55 \mathrm{~cm}$ \\
$C_{5}=502.4 \mathrm{~J} / \mathrm{kg} / \mathrm{K}$ \\
$\rho_{\mathrm{s}}=7.8 \mathrm{gm} / \mathrm{cm}^{2}$ \\
Methane properties \\
$C_{\mathrm{p}}=36 \mathrm{~J} / \mathrm{mol} / \mathrm{K}$ \\
Adsorption properties $(P$ in atm, $T$ in $\mathrm{K}, N$ in $\mathrm{mol} / \mathrm{gm})$ \\
$k_{0}=6.1317$ \\
$k_{1}=-2019.25$ \\
$b_{0}=0.220956$ \\
$b_{1}=29.72837$ \\
\hline
\end{tabular}

which is the ratio of the amount of methane delivered under dynamic conditions over that at isothermal conditions.

$$
\eta=(\text { dynamic } V \text { per } V) /(\text { isothermal } V \operatorname{per} V \text { ). }
$$

A value of unity of $\eta$ is only achievable at an infinitely slow discharge rate. The lowest value of $\eta$ is at adiabatic conditions.

A temperature distribution exists in the cylinder at depletion. Averaging the temperature over the volume of the cylinder provides a single temperature value which indicates the extent of cooling at any given time. The volume average temperature is defined as

$$
<T>=\int_{0}^{L} \int_{0}^{R} \frac{T .2 \pi r \mathrm{~d} r \mathrm{~d} z}{\pi R^{2} L} .
$$

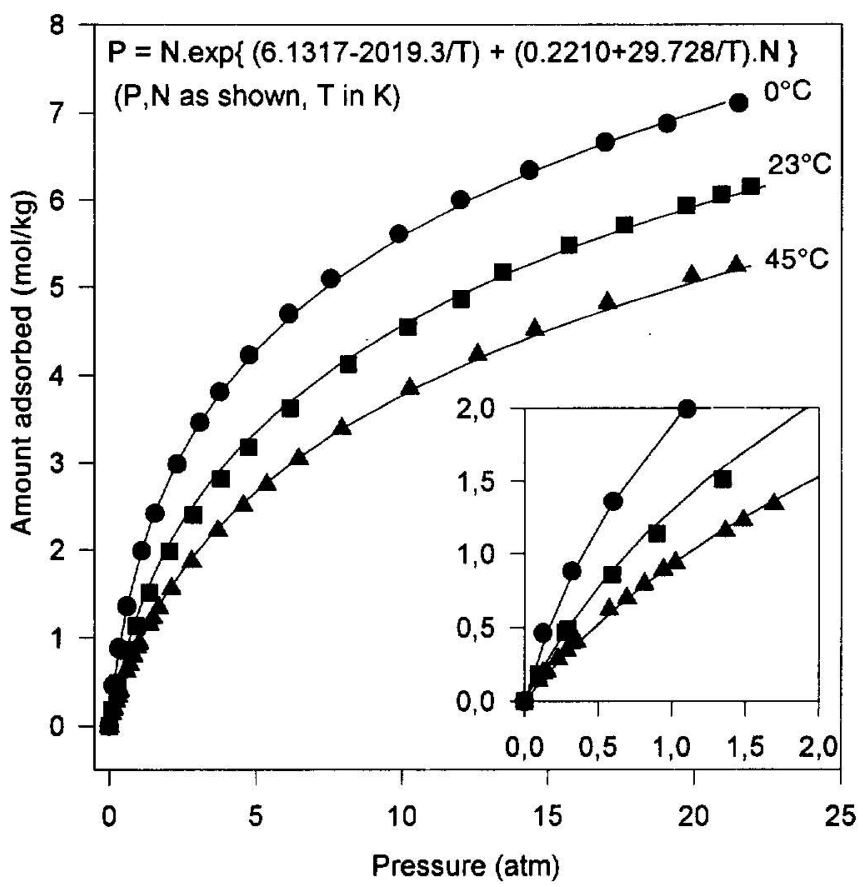

Fig. 3. Methane adsorption isotherms. 
Table 2. Experimental and model results

\begin{tabular}{|c|c|c|c|c|c|}
\hline \multirow[b]{2}{*}{ Cylinder } & \multirow{2}{*}{$\begin{array}{c}\text { Demand } \\
(1 / \mathrm{min})\end{array}$} & \multicolumn{2}{|c|}{ Experimental } & \multicolumn{2}{|c|}{ Model } \\
\hline & & $\Delta T$ & $\eta$ & $\Delta T$ & $\eta$ \\
\hline 1 & 1.03 & -5.3 & 0.92 & -4.8 & 0.85 \\
\hline 1 & 2.01 & -8.9 & 0.94 & -8.4 & 0.85 \\
\hline 1 & 4.53 & -16.1 & 0.85 & -15.7 & 0.81 \\
\hline 1 & 6.72 & -22.8 & 0.81 & -19.8 & 0.78 \\
\hline 1 & 15.01 & -25.7 & 0.75 & -26.5 & 0.73 \\
\hline 2 & 1.04 & -5.7 & 0.95 & -4.9 & 0.86 \\
\hline 2 & 2.09 & -7.2 & 0.94 & -8.9 & 0.87 \\
\hline 2 & 4.54 & -15.8 & 0.85 & -16.1 & 0.84 \\
\hline 2 & 6.45 & -16.8 & 0.80 & -19.8 & 0.81 \\
\hline 2 & 14.02 & -22.9 & 0.78 & -26.5 & 0.76 \\
\hline \multicolumn{6}{|c|}{ Insulated runs } \\
\hline 1 & 4.42 & -24.6 & 0.77 & -27.0 & 0.72 \\
\hline 2 & 4.55 & -24.6 & 0.76 & -28.9 & 0.72 \\
\hline \multicolumn{6}{|c|}{ Runs with perforated tube inserted } \\
\hline 1 & 4.55 & -15.4 & 0.87 & & \\
\hline 1 & 6.67 & -18.5 & 0.86 & & \\
\hline 1 & 14.98 & -26.7 & 0.78 & & \\
\hline
\end{tabular}

\section{RESULTS AND DISCUSSIONS}

Table 2 lists the results from experiments and the model as dynamic efficiency and volume average temperature drop. The highest measured volume average temperature drop is about $29^{\circ} \mathrm{C}$. The lowest measured efficiency is 0.75 , corresponding to $25 \%$ loss of isothermal capacity due to cooling during desorption. Model results are close to data in general. The discrepancy between the model and the data is lower at higher flow rates.

\section{Pressure and temperature transients during $A N G$ discharge}

Since it is not possible to tabulate the large volume of data, the changes that occur in an ANG cylinder during discharge are shown only for a typical run with cylinder 1 at $6.7 \mathrm{l} / \mathrm{min}$ demand rate. This discharge rate corresponds to one-quarter of the energy demand of a subcompact car

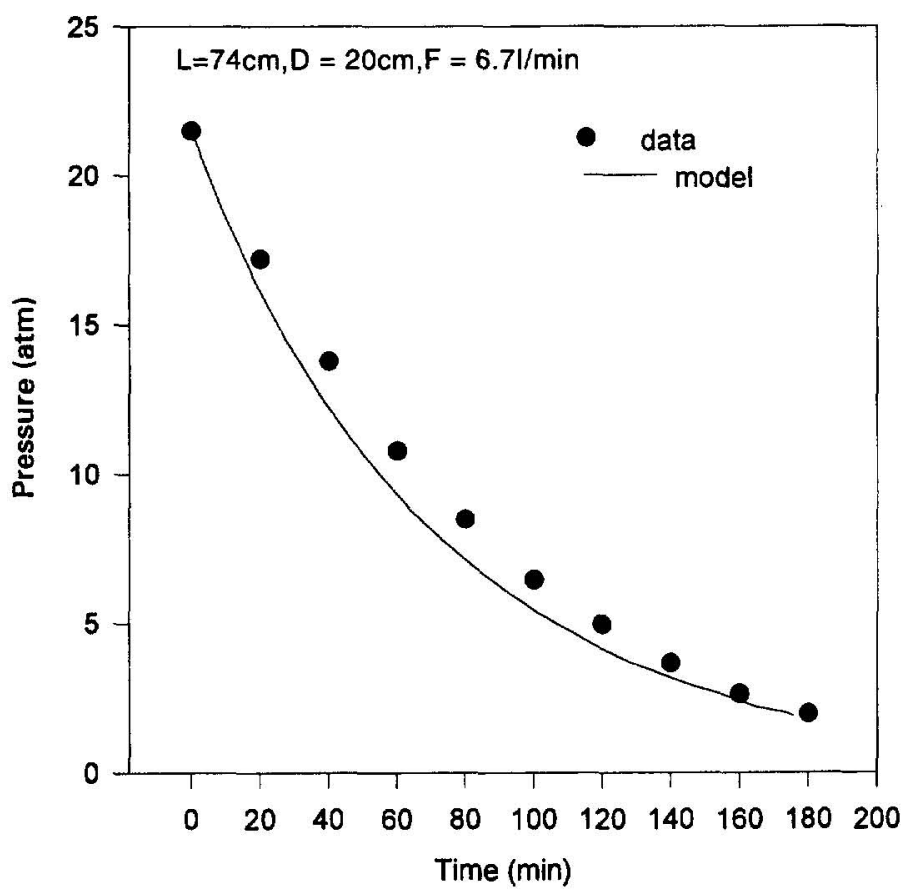

Fig. 4. Typical pressure transient in an ANG cylinder during discharge. 


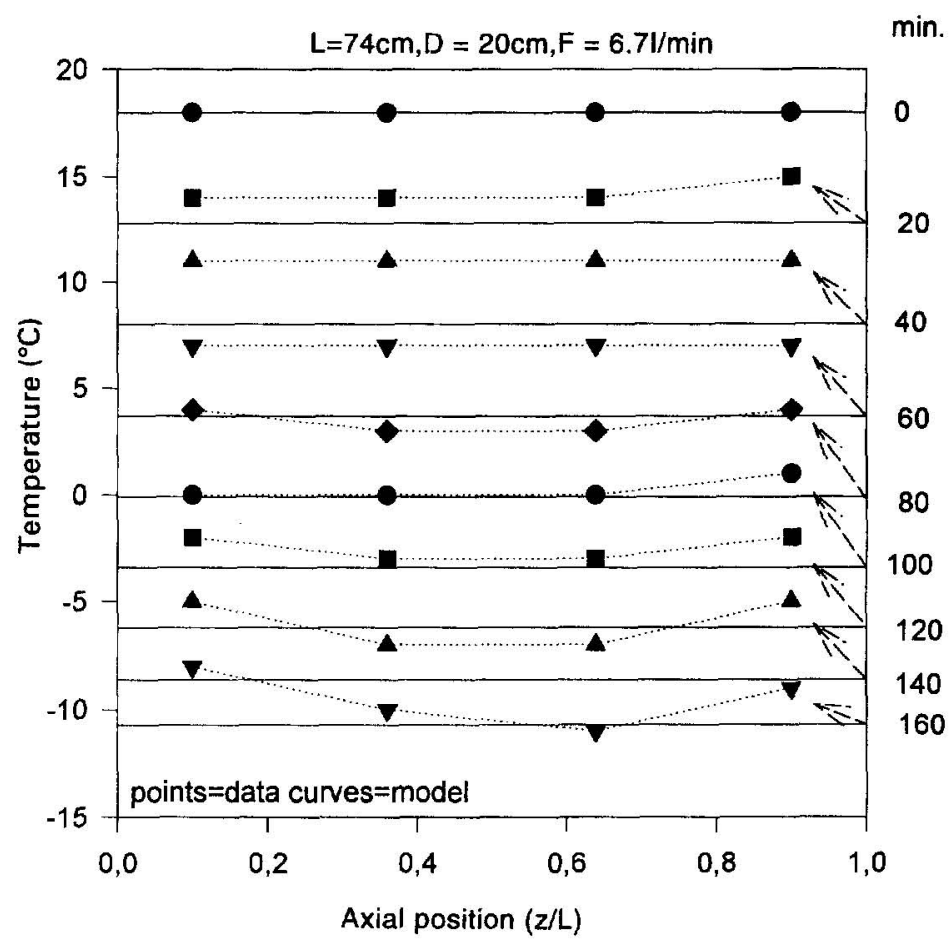

Fig. 5. Axial temperature profiles at the center of an ANG cylinder as a function of time during discharge.

traveling at cruising speed. For this specific experiment, the volume average temperature drop was $22.8^{\circ} \mathrm{C}$, resulting in a $19 \%$ loss of capacity. Corresponding values by simulation are $19.8^{\circ} \mathrm{C}$ and $22 \%$, respectively.

The pressure history is shown in Fig. 4. The pressure variation with time is nonlinear, since it corresponds to the tranformation of a fixed demand rate by the nonlinear isotherm relation. The discrepancy between the model and the experiment at intermediate times is probably caused by the linear compensation for gas contribution in the accumulation term in the model. The error introduced into the dynamic efficiency is fairly small, the experimental and model efficiencies are 0.81 and 0.78 , respectively, for the case displayed in Fig. 4.

Axial temperature variation was neglected in the model. Figure 5 shows axial temperature data at the centerline, where the largest temperature drop occurs. The highest temperature variation in the axial direction is $3^{\circ} \mathrm{C}$ at depletion. The variation is lower at larger radial positions, approaching zero at the cylinder wall. As evident in the figure, some axial variation in temperature does occur. We suspect that most of this temperature variation in data is caused by the 'end-effects', the cylinder bottom and top have considerable thermal capacity in their hardware, such as the bulkhead walls, the cylinder valve, the cylinder foot, etc. These were not included in the model.

Figure 6 for radial temperature distribution with time is an excellent illustration of the impact of heat of adsorption on ANG discharge. The small dispersion in data on the profiles is the result of thermocouples being placed at different axial positions. There are several aspects in Fig. 6 that need to be noted:

At depletion, the center of the cylinder cools to $-11^{\circ} \mathrm{C}$, corresponding to a $29^{\circ} \mathrm{C}$ temperature drop. The volume-averaged temperature drop is $22.8^{\circ} \mathrm{C}$ for this run. These are fairly large temperature variations, which cause a significant shift in the adsorption isotherms.

The cylinder wall is $8^{\circ} \mathrm{C}$ at depletion. The temperature is $6^{\circ} \mathrm{C}$ at the thermocouple closest to the wall. The temperature difference is about $18^{\circ} \mathrm{C}$ between the wall and the centerline, which indicates the importance of the energy supplied by the steel wall and by natural convection outside the wall. The profiles would be flat if desorption was adiabatic with all desorption energy supplied by the carbon.

The overall temperature gradient is approximately $1.8^{\circ} \mathrm{C} / \mathrm{cm}$ in the radial direction at depletion. The maximum axial temperature gradient is $0.07^{\circ} \mathrm{C} / \mathrm{cm}$ from Fig. 5 at the same instant. Although 


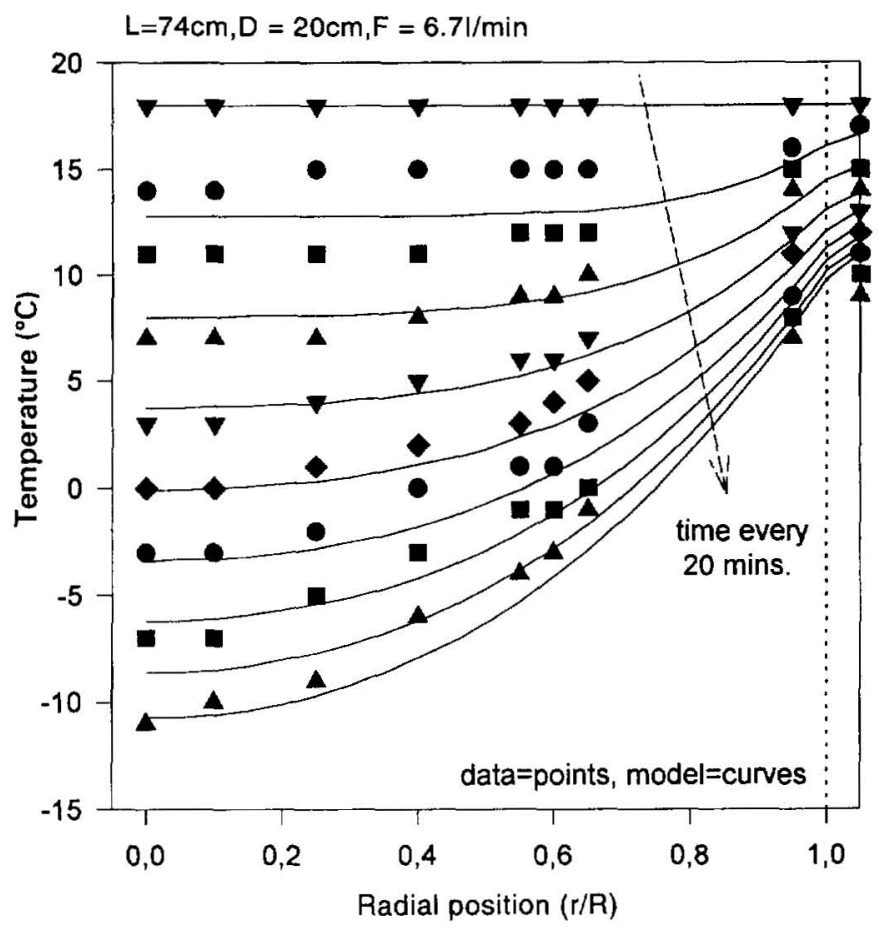

Fig. 6. Radial temperature profiles in an ANG cylinder as a function of time during discharge.

it is not completely correct to compare the two gradients due to the system geometry, it is clearly evident from these numbers that even the slow flow of methane in the axial direction is beneficial for energy transfer, smoothing the temperature variation in the axial direction.

The model results follow data fairly closely in Fig. 6. Wall temperature shows the largest discrepancy with the model, showing higher temperatures than experimentally measured. Many explanations can be offered but none can be singled out with confidence: neglection of convective

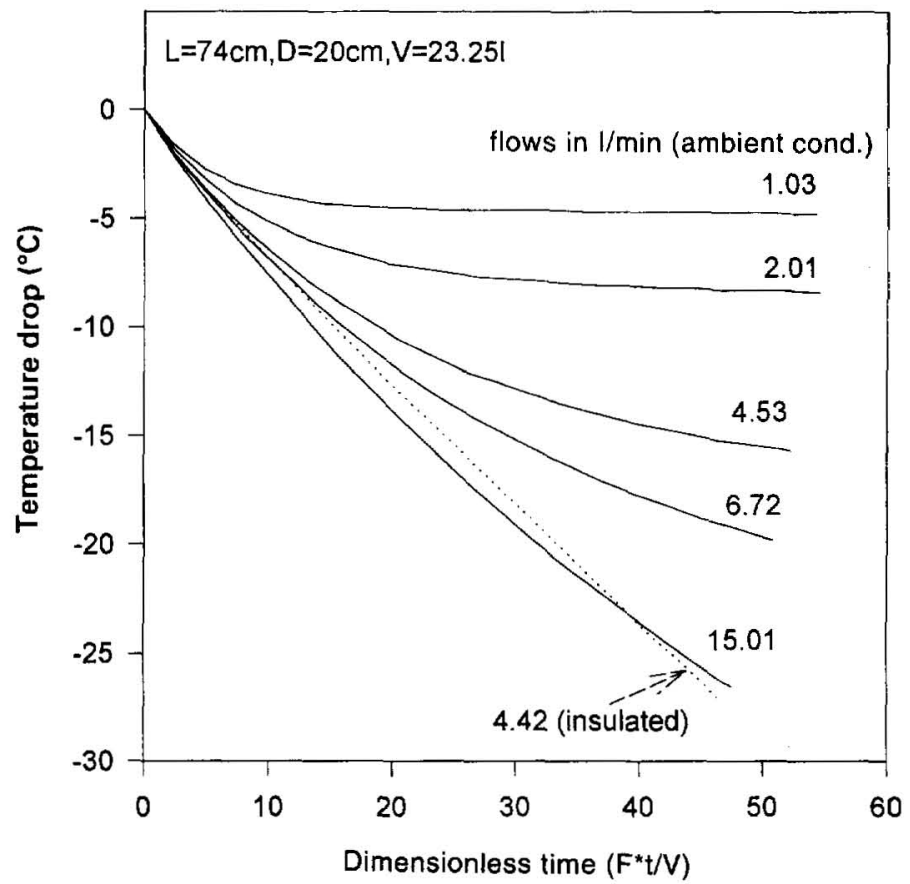

Fig. 7. Effect of discharge rate on volume average temperature drop in an ANG cylinder during discharge. 


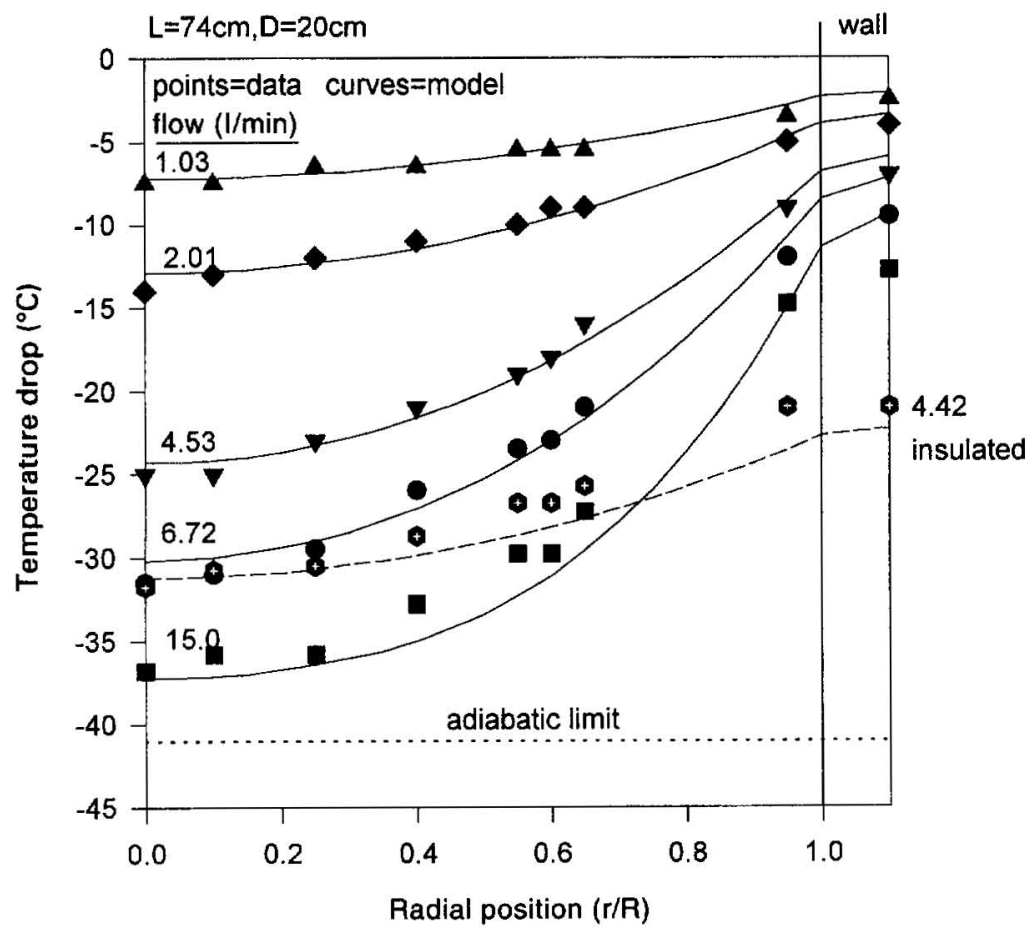

Fig. 8. Radial temperature profiles at depletion in an ANG cylinder.

transfer inside the wall, explosion room conditions not being 'truely' natural convection, etc. We conclude from Fig. 6 that the model captures the essence of ANG desorption dynamics, although somewhat more accuracy is desirable.

\section{Effect of demand rate and conditions outside the cylinders}

The temperature in ANG cylinders is predominantly determined by the demand rate. Figure 7 shows simulation results for volume average temperature drop with dimensionless time. It is generally argued that ANG systems can be engineered to remedy the impact of heat of adsorption. Thus, most literature cites the isothermal capacities, while it is not possible to operate a real system under isothermal conditions. Isothermal operation is only possible at infinitely slow discharge, which, of course, is impossible in a real operation. The lowest flow rate in Fig. 7 might be the closest to a 'slow' discharge. The temperature drop is $5.3^{\circ} \mathrm{C}$, corresponding to an $8 \%$ capacity loss and this is for an unrealistically slow experiment which lasted more than $20 \mathrm{hr}$. It is hard to envision any engineering remedy that can lower the impact of heat of adsorption below this level.

The lowest flow case reaches steady-state where the energy of desorption is completely supplied by natural convection outside the cylinder once enough temperature difference is established. The temperature drop increases with increasing flow as expected. The transient for the highest flow rate in Fig. 7 has some curvature, indicating that there is some energy transferred into the system by natural convection.

It is interesting to note that the temperature with the highest flow is lower than the insulated case at intermediate times. There is not enough time even to utilize the thermal energy stored in the cylinder wall at a high flow rate. This is displayed clearly in Fig. 8, where the radial temperature profiles are shown at depletion. As flow rate increases, the temperature gradient in the cylinder becomes larger, although the wall temperature drops further.

The overall effect of conditions outside the cylinder is demonstrated in Fig. 9, where a $25^{\circ} \mathrm{C}$ temperature drop is observed with insulation compared to about $15^{\circ} \mathrm{C}$ without insulation at the same discharge rate. Another indication of the contribution of convective energy input to the cylinder is clearly shown in Fig. 8, where the temperature profile with insulation is much flatter than others.

It should be noted that the runs with insulation do not correspond to true 'adiabatic desorption', 


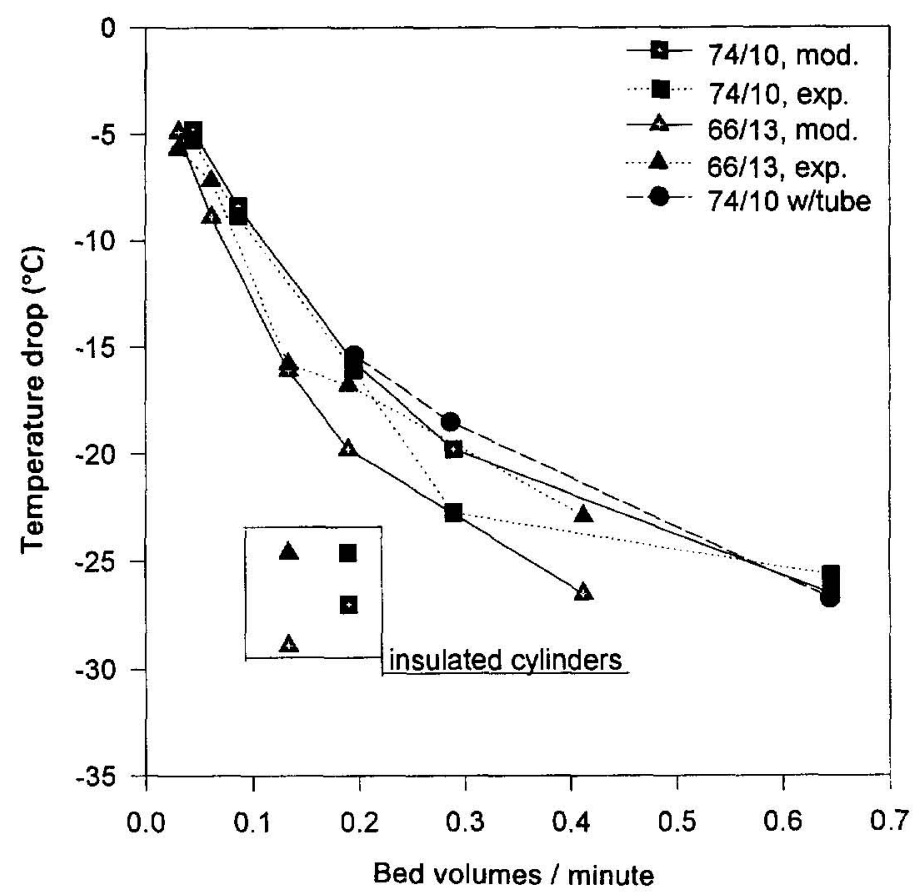

Fig. 9. Effect of discharge rate on volume average temperature drop at depletion in ANG cylinders ( $L / D$ ratios as shown).

where all desorption energy is supplied by the sensible heat of the adsorbent. The thermal energy stored in the cylinder wall is still being utilized in the experiments with insulation, as evident from the small but perceptible curvature of the temperature profile in Fig. 8. The cylinder wall is an important energy source in ANG systems, justifying the care taken in order not to bias the dynamics by using lab-scale cylinders in these experiments.

The central locations with the highest flow rate approach adiabatic condition with a $37^{\circ} \mathrm{C}$ drop

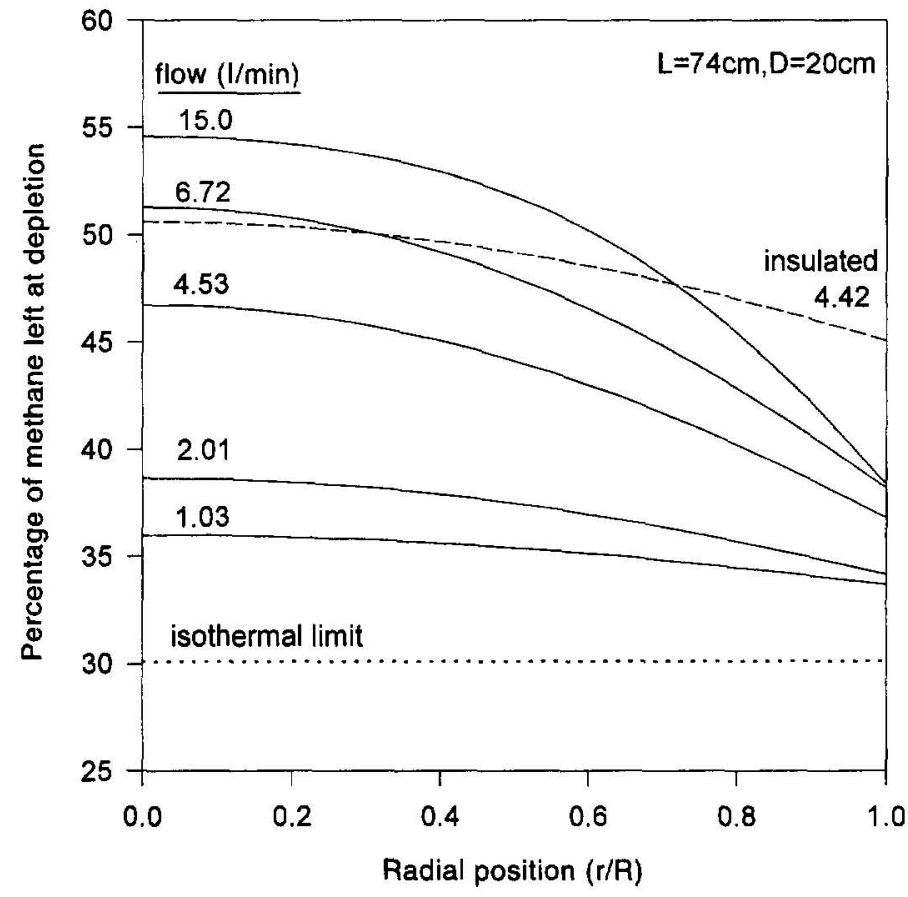

Fig. 10. Radial profile of residual amount of methane left in ANG cylinders at depletion. 


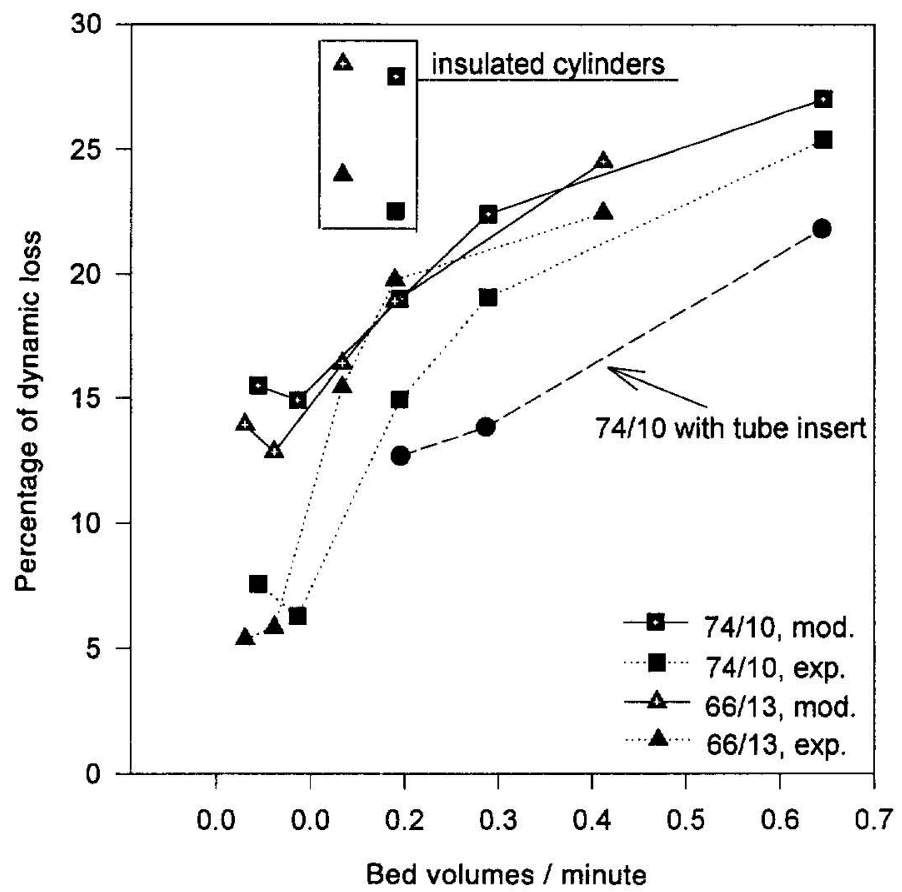

Fig. 11. Dynamic losses in ANG discharge as a function of discharge rate ( $L / D$ ratios as shown).

in temperature shown in Fig. 8. The adiabatic temperature drop for these experiments from theoretical considerations [6] is about $41^{\circ} \mathrm{C}$.

The result of large temperature drop is a higher amount of methane retained in the cylinder at depletion. Figure 10 shows the profiles of residual methane left in the cylinder at depletion as a percentage of the amount at charge conditions. The residual methane is about $30 \%$ under isothermal conditions, whereas it is $36 \%$ at the center, even at the lowest flow rate. The residual is as high as $54 \%$ at the centerline at the highest flow. The shapes of residual methane profiles are inversely related to the temperature profiles shown in Fig. 8 by the isotherm relation.

Figure 11 shows the overall impact of heat of adsorption on ANG systems as a percentage loss to dynamics. The highest measured value is a $25 \%$ reduction from the isothermal capacity. The data and the model in the figure approach a plateau with increasing discharge rate. The plateau level is determined by adiabatic conditions. In general, the model overpredicts the dynamic losses, especially at low demand rates. Furthermore, the data show a wider difference than the model between the two cylinder geometries. At the flow regimes likely to be encountered in a vehicle application, the dynamic loss is about $15-20 \%$, depending on the $L / D$ ratio of the cylinder.

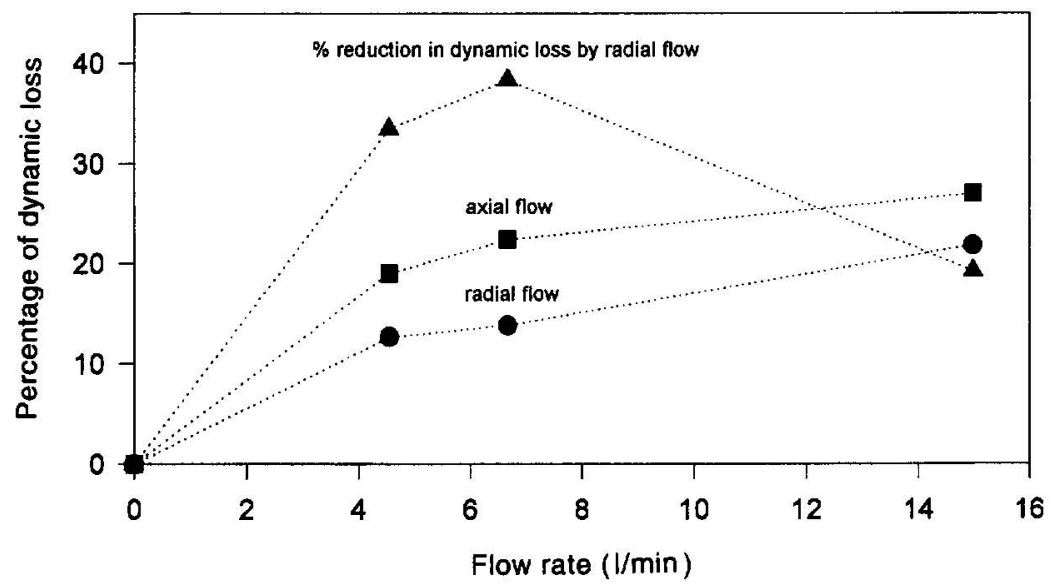

Fig. 12. Experimental data for the effect of flow direction during discharge of ANG cylinders. 
It should be noted that the activated carbon in these cylinders was not of very high quality. The isothermal performance is about $60 \mathrm{~V}$ per $V$, whereas better carbons can provide as high as 90 $V$ per $V$ under similar conditions. Conversely, a better carbon does not necessarily result in a better performance under dynamic conditions. The dynamic losses will increase with increasing performance. This is simply a result of more methane being desorbed per carbon weight. It is possible that a low-quality carbon may perform better than a higher-quality carbon under dynamic conditions.

The dynamic losses are limited by adiabatic desorption but, as can be seen in this study, ANG systems during realistic discharge cycles do not operate under adiabatic conditions. Therefore, it is not possible to simply assess the utility of a carbon by the performance at the two limiting regimes: isothermal and adiabatic. The system must be tested under realistic conditions, as done in this study.

\section{THE EFFECT OF FLOW DIRECTION}

It is not possible to operate an ANG system under isothermal conditions. During the discharge cycle, an important energy source is the cylinder wall and heat transfer from the surroundings through the cylinder wall. The poor conductivity of packed carbon particles presents a large resistance to heat transfer and prevents effective utilization of the energy input from the cylinder wall. The engineering solution is obvious from figures showing the gradients that develop in an ANG cylinder: increase the energy supply to the central region of the cylinder.

There are some suggested remedies in the literature to moderate the temperature fluctuations in ANG cylinders. These mainly concentrate on two approaches: (1) increasing the conductivity, i.e. including steel shavings [13]; and (2) increasing the thermal capacity, i.e. thermal energy storage (TES) capsules suggested by IGT researchers [21]. Both approaches suffer from the fact that they occupy space in the storage vessel, reducing the volume available for storage. These remedies are also prone to mechanical difficulties, such as segregation of these foreign particles in a cylinder over time. Furthermore, TES can only be designed for a specific ambient temperature, while ANG systems are expected to operate under widely varying conditions.

An alternative approach was tested in this study to enhance the energy transfer to the central region of the cylinder. A perforated $3 / 8$ in. tube was welded to the cylinder valve, which was then inserted at the centerline of the carbon-packed cylinder. The amount of carbon in the cylinder was unchanged, it was quite easy to push a fairly small tube into the carbon-packed cylinder. The tube acts like a gas collector at the centerline, effectively changing the flow direction from axial to radial. Therefore, gas desorbed closer to the cylinder wall is used as a medium to transfer energy to the central region. Since methane has a fairly low heat capacity, being a gaseous compound, the benefits of this approach may not be readily apparent. The real advantage stems from the fact that the energy transfer mechanism is not completely based on the sensible heat. When adsorption is of concern, the energy content of a methane molecule in the gas phase is the sum of its sensible heat and the heat of adsorption, the latter being much larger. Therefore, the energy transfer can be much higher than is possible with only the sensible heat. Furthermore, this approach circumvents all three problems cited above for other remedies, such as TES capsules:

The space occupied by the perforated tube is very small. Also, the total carbon weight is unchanged; there is no reduction in the amount of methane in the cylinder at charge conditions with the tube insert.

The segregation problems of 'composite' beds do not exist for a permanently fixed tube.

The benefits of the tube insert are realized regardless of the ambient conditions, they are not condition specific.

The result of changing flow direction from axial to radial is a more uniform temperature distribution in the cylinders. That is, lower wall temperatures, but higher temperatures at the central region of the ANG cylinders. The modification of temperature profiles lowers the dynamic loss, as shown in Fig. 12 which displays experimental results; we did not attempt to model the radial flow case. The radial flow with the insert consistently shows lower dynamic loss than axial flow under the same experimental conditions. The reduction in loss, or the difference between the two curves, peaks at some intermediate demand rate. The maximum benefit is observed at about $71 / \mathrm{min}$ 
demand rate, where the loss is $22 \%$ with axial flow compared to $12 \%$ with radial flow, corresponding to a reduction of about $40 \%$ in dynamic losses.

The shape of the reduction in dynamic loss in Fig. 12 needs further consideration. At infinitely slow discharge rate, the flow direction does not matter, since the system is isothermal. At infinitely fast discharge rates (adiabatic conditions) the flow direction also does not matter, since all desorption energy is supplied by the adsorbent. The flow direction only has an impact at intermediate flow rates, as displayed in Fig. 12. The location of the maximum benefit depends on other physical parameters, such as cylinder geometry, packing conductivity, etc. It is certain that there will be a maximum at some intermediate flow between the isothermal and abiabatic extremes. Matching the flow at the maximum with the expected demand rate for a specific ANG cylinder is a simple engineering problem. We did not do any detailed optimization study, it is by chance that the maximum in Fig. 12 at 7 1/min happens to be the expected average demand rate for the cylinders used in this work.

\section{CONCLUSIONS}

The purpose of this study was to assess the impact of the heat of adsorption on ANG systems under realistic discharge conditions. Using extensive experimental data collected with real ANG cylinders and a fairly simple model, we have demontrated the following:

It is not possible to operate an ANG system under isothermal conditions. Any finite discharge rate will result in a temperature drop. At the smallest controllable discharge rate, the observed temperature drop is about $5^{\circ} \mathrm{C}$, resulting in an $8 \%$ loss of capacity.

The temperature drop can be very substantial, the maximum measured was $37^{\circ} \mathrm{C}$ at the highest demand rate used. This results in the residual methane at depletion increasing to $54 \%$ of the charge amount, compared to only $30 \%$ for the isothermal operation. The overall loss to dynamics at the highest discharge rate is $29 \%$.

Under realistic conditions of a vehicle application, the dynamic loss is expected to be $15-20 \%$.

At the other extreme, ANG discharge is not an adiabatic operation. The thermal capacity of the cylinder wall is an important energy source and external convective heat transfer can supply a significant amount of energy. The main obstacle in utilizing these energy sources is the poor thermal conductivity of packed carbon.

A simple, yet effective, remedy to increase energy transfer to the central region of ANG cylinders is changing the flow direction from axial to radial. This was accomplished by a perforated tube inserted at the centerline, which acts as a collector for the exiting gas. Unlike other suggested remedies to moderate the impact of heat of adsorption, (1) the tube insert does not significantly reduce precious storage space, (2) it is easy and inexpensive to implement and (3) it moderates the temperature drop under any ambient condition.

The dynamic loss is reduced from 22 to $12 \%$ with the tube insert at the most pertinent flow condition. This represents a $40 \%$ reduction in loss.

Acknowledgements - This work was partly sponsored by the Academic Challenge Program of the State of Ohio.

\section{REFERENCES}

1. C. F. Blazek, Use of alternative fueled vehicles. Presented at Kentucky Air and Waste Management Association NO Control Workshop, 24-25 January 1990.

2. O. Talu, An overview of adsorptive storage of natural gas. In Fundamentals of Adsorption (edited by M. Suzuki), p.655 (1992).

3. Z. Tan and K. E. Gubbins, Adsorption in carbon micropores at supercritical temperatures. J. Phys. Chem. 94, 6061 (1988).

4. M. J. Bojan, R. Van Slooten and W. Steele, Computer simulation studies of the storage of methane in microporous carbons. Sep. Sci. Technol. 27, 1837 (1992)

5. K. R. Matranga, A. Stella, A. L. Myers and E. D. Glandt, Molecular simulation of adsorbed natural gas. Sep. Sci. Technol. 27, 1825 (1992).

6. K. R. Matranga, A. L. Myers and E. D. Glandt, storage of natural gas by adsorption on activated carbon. Chem. Engng Sci. 47, 1569 (1992).

7. S. S. Barton, J. R. Dacey and D. P. Quinn, High-pressure adsorption of methane on porous carbons. Fundamentals of Adsorption (edited by A. L. Myers and G. Belfort), United Engineering Trustees, p.65 (1984). 
8. S. S. Barton, J. A. Holland and D.F. Quinn, Modification of microporous carbon for methane storage. Fundamensals of Adsorption (edited by I. Liapis), United Engineering Trustees, p.99 (1986).

9. D. F. Quinn and J. A. MacDonald, Natural gas storage. Carbon 30, 1097 (1992).

10. L. Czepirski, Some aspects of a sorbent containing storage system for natural gas. Indian J. Technol. 29, 266 (1991).

11. B. Buczek and L. Czerpirski, Improvement of methane storage capacity for active carbons. Adsorp. Sci. Technol. 4, 217 (1987).

12. R. Chahine and T. K. Bose, Measurement of physical adsorption of gases at high pressure. Proc. Int. Symp. Measurements, Props and Utilization of Natural Gas, Montreal, Quebec (1987).

13. N. Sangani, Desorption dynamics of adsorptive gas storage systems, MS. Thesis, Cleveland State University, Cleveland (1990).

14. J. P. Barbosa Mota, Modelisation des Tranferts Couples en Milieux Poreux, Etude du Stockage de Gaz Naturel par Adsorption et de la Convection Naturelle en Espace poreux Annulaire, Dissertation, Institut National Polytechnique de Lorraine (1995).

15. J. P. Barbosa Mota, E. Saatdjian, D. Tondeur and A. E. Rodrigues, A simulation model of a high-capacity methane adsorptive storage system. Adsorption 1, 17 (1995).

16. K. J. Chang, Dynamic performance of ANG storage systems, MS. Thesis, Cleveland State University, Cleveland (1994).

17. W. J. Jasionowski, A. J. Tiller, J. A. Fata, J. M. Arnold, S. W. Gauthier and Y. A. Shikari, Charge/discharge characteristics of high-capacity methane adsorption storage systems. Int. Gas Res. Conf., Tokyo, Japan (1989).

18. R. J. Remick and A. J. Tiller, Advanced methods for low-pressure storage of CNG. Proc. Non-petroleum Vehicular Fuels Symp., Chicago, Illinois (1985).

19. R. T. Biederman and C. F. Blazek, Economic Analysis of Low-pressure Natural Gas Vehicle Storage Technology, Topical Report, GRI Cont. No. 5086-530-1365, April (1990).

20. R. Chahine and T. K. Bose, Adsorption storage of gas fuels on activated carbons. Presentation at the AlChE Annual meeting, San Francisco (1994).

21. W. H. McAdams, Heat Transmission. McGraw-Hill, New York (1954).

22. O. Talu, J. Li and A. L. Myers, Activity coefficients of adsorbed mixtures. Adsorption 1, 103 (1995).

23. V. G Jenson and G. V. Jeffreys, Mathematical Methods in Chemical Engineering. Academic Press, London (1977) 